A N N A L E S Annales de Bretagne et des Pays de l'Ouest

Anjou. Maine. Poitou-Charente. Touraine

109-4 | 2002

Les étrangers dans l'Ouest de la France (XVIII $-\mathrm{XXX}^{\mathrm{e}}$ siècle)

\title{
Prisonniers de guerre et civils internés allemands dans le département de la Vienne (1945-1948)
}

\section{Loïc Rondeau}

\section{(2) OpenEdition}

Journals

Édition électronique

URL : http://journals.openedition.org/abpo/1533

DOI : $10.4000 /$ abpo.1533

ISBN : 978-2-7535-1488-1

ISSN : 2108-6443

Éditeur

Presses universitaires de Rennes

Édition imprimée

Date de publication : 20 décembre 2002

Pagination : $217-227$

ISBN : 978-2-86847-794-1

ISSN : 0399-0826

Référence électronique

Loïc Rondeau, "Prisonniers de guerre et civils internés allemands dans le département de la Vienne (1945-1948) », Annales de Bretagne et des Pays de l'Ouest [En ligne], 109-4 | 2002, mis en ligne le 20 décembre 2004, consulté le 01 mai 2019. URL : http://journals.openedition.org/abpo/1533 ; DOI $10.4000 / a b p o .1533$ 


\title{
Prisonniers de guerre et civils internés allemands dans le département de la Vienne (1945-1948)
}

\author{
LoÏc RONDEAU \\ Doctorant en histoire contemporaine, Université de Poitiers
}

Lorsque l'on parle de l'emploi des prisonniers de guerre allemands en France après la Seconde Guerre mondiale, nous pensons souvent au déminage des côtes. Or, la présence allemande fut également importante dans tous les départements. D'après le ministère des Affaires étrangères, 1035000 prisonniers sont sur le territoire français en $1945^{1}$.

Le département de la Vienne présente l'intérêt d'une présence allemande sous deux aspects ${ }^{2}$. Sont internés au camp de la Chauvinerie, fonctionnant de février à novembre 1945, des milliers de personnes, hommes, femmes et enfants, venant souvent de l'Est de la France, à mesure que les Alliés avancent. Il n'est pas facile de déterminer un " type " d'interné pour ce camp. Nous aborderons brièvement l'historique du camp et son fonctionnement, avant de traiter des conditions de vie difficiles des internés, qui, ajoutées à l'état physique faible des arrivants, entraînent une forte mortalité. Nous verrons enfin que l'histoire de la Chauvinerie est marquée par ce qu'on a appelé le " scandale du camp ", avec des abus et des détournements de biens.

L'autre forme de la présence allemande est symbolisée par les « commandos ". En septembre 1946, près de 4000 prisonniers de guerre sont employés, notamment dans l'agriculture qui manque alors de main-d'œuvre. Mais, les Français sont-ils prêts à accepter une présence allemande, après quatre années d'occupation? Après avoir vu les modalités d'emploi, nous étudierons l'utilisation qui a été faite de ces prisonniers, et enfin leurs rapports avec les Français et les autorités.

1. Arch. nat. BB18 3693(1), rapport du ministère des Affaires étrangères au ministère de la Justice, 17 décembre 1948.

2. Cette contribution présente le résultat de recherches effectuées dans le cadre de notre thèse portant sur le département de la Vienne à la Libération, sous la direction de M. Gérard Baal. 


\section{Le Camp de la Chauvinerie}

\section{Installation et fonctionnement du camp}

À partir de la mi-février 1945, les autorités utilisent le camp de la Chauvinerie à Poitiers, pour les internés allemands, et ce jusqu'au début de novembre de la même année. Ils viennent, pour la plupart, des régions de l'est de la France libérées par les Alliés, et sont considérés comme " suspects ". Ce sont parfois des familles entières qui sont conduites à Poitiers. Sur la première liste des internés établie fin février se trouvent 670 hommes, 201 femmes, 65 enfants de 3 à 14 ans, et 20 de moins de trois ans. Déjà, six hommes décèdent entre le 16 et le 25 février. Sur les vingt enfants de moins de trois ans, quinze sont nés en Moselle. Puis arrivent des personnes venues d'autres camps et qui avaient été internées pendant la guerre par les Allemands ou par les autorités françaises, et qui attendent leur libération. La presse a comparé plus tard ce camp à une "gare de triage $^{3}$ ». Un rapport bi-mensuel rédigé par le directeur donne les effectifs du camp. Le nombre d'internés le plus élevé est relevé dans le rapport du 31 juillet 1945, date à laquelle 3913 personnes sont internées, dont 1898 hommes, 1461 femmes, 525 enfants de moins de 14 ans, et 25 prisonniers de droit commun ${ }^{4}$.

Pour chaque interné, une fiche était établie à l'entrée dans le camp. Ces nombreuses fiches, 4871 au total (2253 hommes, 1913 femmes, 539 enfants de 3 à 14 ans, et 166 enfants de moins de trois ans), sont conservées aux archives départementales de la Vienne, et nous ont permis de constater la diversité des internés. En effet, nous avons pu relever des cas de personnes qui avaient subi la répression du régime nazi, pour diverses raisons, du simple vol au refus d'obéissance, en passant par l'opposition politique. Il nous est difficile de connaître le cheminement particulier de chaque interné, car le motif d'internement est très rarement noté sur les fiches. Nous connaissons toutefois les camps d'où ils arrivent : le Strutfof (721 femmes et 438 hommes), Dôle (599 femmes et 323 hommes), Écrouves (283 femmes et 655 hommes), Noé (442 femmes et 157 hommes) sont les camps de provenance les plus importants ${ }^{5}$.

Comme tous les camps, celui de la Chauvinerie a son règlement, rédigé en 25 articles. Une baraque ne peut accueillir plus de cent internés, "l'aménagement comprend des couchettes tendues de grillage sur lequel il est répandu de la paille libre ", et " les couvertures appartiennent aux internés " (article 2). Dans chaque baraque, un responsable parlant français est désigné par le chef du camp (article 3). La vie quotidienne est rythmée par le réveil, les appels, la nourriture, les récréations, et le travail. Le réveil "varie suivant les saisons " (article 4). Les appels se font le matin, à $12 \mathrm{~h} 30$ et à $20 \mathrm{~h} 30$ (article 5). À $21 \mathrm{~h} 30$, on ferme les baraques et on éteint les lumiè-

3. La Nouvelle République, 27 janvier 1949.

4. Arch. nat. F7 14970, rapport bi-mensuel du directeur du camp, 31 juillet 1945.

5. Arch. dép. de la Vienne, 104W167, fiches des internés. 
res (article 6). "Le genre de rations est fixé par une instruction ministérielle; le taux en est déterminé par le préfet en tenant compte des ressources du ravitaillement général local " (article 7). Les mères de familles et leurs enfants peuvent rester dans la cour d'appel, du matin à 19 heures; les autres femmes et les hommes de 9 heures à 11 heures et de 14 heures à 19 heures (article 16).

Les prisonniers peuvent être utilisés pour des travaux à l'extérieur du camp. Ils doivent être volontaires, et sont soumis à un contrat de travail. Ainsi plusieurs dizaines d'internés participent à la reconstruction de la gare de Poitiers, bombardée en juin 1944 par les Alliés. Le chef du camp assure la surveillance grâce à des gardiens armés qui ne sont ni logés, ni nourris par l'employeur. En théorie, ils ne peuvent pas travailler pour des particuliers. Ainsi, lorsque $\mathrm{M}^{\mathrm{me}} \mathrm{C} . \mathrm{S} .{ }^{6}$, qui possède un bar et dont le mari est malade, demande une internée pour l'aider, le colonel Blanchard, responsable du camp, donne son accord, mais le préfet refuse. Ce dernier ne sera pas toujours écouté...

\section{L'état sanitaire des internés}

La présence des Allemands est marquée par des conditions d'hygiène alarmantes. Dès le premier mois, le préfet signale que " chez les hommes, la plupart réformés du service militaire ou inaptes, l'état de santé laisse beaucoup à désirer ". Neuf décès sont déjà enregistrés au 5 mars. Le 13 avril, le rapport mensuel du préfet relève l'état de santé "franchement mauvais pour les hommes ". Le mois suivant, 33 enfants décèdent. Une note de la $1^{\text {re }}$ division au chef de cabinet de la préfecture, datée du 11 juin 1945, précise que le ministère de l'Intérieur a été tenu au courant de cette situation, " par lettres des 30 mai, $1^{\text {er }}$ juin et 8 juin 1945 ", selon laquelle les internés fragiles "fautes d'aliments et de soins appropriés, sont tous appelés à disparaître".

\section{Pour le directeur du camp,}

" dans les convois qui arrivent au camp figurent des personnes dont l'état physique lamentable aurait sans doute pu les dispenser d'être internées aussi loin de chez elles. Il n'apparaît pas que ces personnes puissent présenter un danger national, par contre elles transforment la silhouette du camp d'internement en un vaste hôpital ${ }^{7}$."

Le nombre de décès est difficile à connaître précisément. Nous avons cependant deux chiffres qui nous donnent une idée de l'importance de la mortalité. D'après les rapports bi-mensuels du directeur du camp, il y aurait eu 265 décès $^{8}$ (dont 148 hommes, 51 femmes et 66 enfants de moins de

6. Nous utilisons les initiales afin de respecter la vie privée des personnes.

7. Arch. nat. F7 14970, rapport mensuel du chef du camp à l'Inspection générale des camps, $1^{\mathrm{er}}$ mai 1945.

8. Arch. nat. F7 14970, rapports bi-mensuels du directeur du camp à l'Inspection générale des camps, du 15 mars au 31 octobre 1945. 
14 ans). D'après les fiches personnelles conservées aux archives départementales de la Vienne, nous avons pu relever 241 fois la mention " décédé " sur les fiches ${ }^{9}$. Sont concernés surtout les personnes âgées et les enfants en bas âge. Deux raisons essentielles sont avancées pour expliquer cette mortalité : l'état faible de beaucoup d'internés avant même leur entrée dans le camp, mais également les conditions de vie à l'intérieur même du camp. Malgré toutes les alertes adressées au gouvernement, celui-ci ne réagit qu'en septembre 1945, lorsqu'éclate le "scandale du camp ".

\section{Le « scandale du camp»}

En septembre 1945, une enquête à l'intérieur du camp va révéler les abus et détournements que connaît le camp. Sans pouvoir ici développer les faits dans le détail, nous pouvons en retenir trois, dénoncés par le commissaire de police judiciaire. Tout d'abord, des échanges ont lieu entre gardiens et internés. Par exemple, une montre et deux robes en provenance du camp sont retrouvées chez la surveillante en chef du camp, lors d'une perquisition. Trois autres surveillantes avouent également participer au trafic.

Ensuite, le gestionnaire est mis en cause pour mauvaise gestion alimentaire : la comptabilité enregistre des quantités d'aliments qui n'arrivent pas aux fourneaux. Le commissaire en tire la conclusion que « les denrées portées en comptabilité au camp ne correspondaient pas aux denrées servies aux rationnaires, ce qui permet de penser qu'une partie de ces denrées a été détournée par les agents du camp ${ }^{10}$ ". Les chiffres qu'il trouve dans les documents du camp accréditent son hypothèse. D'après le livre de compte, la valeur des marchandises est de 262633,01 francs. Or, le stock existant est d'une valeur de 230223,39 francs, soit une différence de 32 409,62 francs.

Enfin, des objets appartenant aux internés sont saisis et distribués par le chef du personnel. La différence est importante entre les objets déclarés par les internés et les récapitulatifs qui arrivent à la préfecture. Le commissaire de police judiciaire a pu remarquer qu'à l'arrivée du camp, l'inventaire n'était dressé que pour les objets de valeur, conservés à la trésorerie du camp, où il serait facile d'entrer et sortir. Pour les vêtements, chaussures et tissus, aucun inventaire n'est dressé, et ils sont parfois distribués aux gardiens et surveillantes. Ainsi, lorsqu'un ancien interné demandera en 1948 la restitution d'une couverture, de deux costumes, de deux paires de chaussures, de deux chemises, d'une robe de chambre, d'un bracelet-montre, d'un réveil, de 150 dollars et de 5 francs suisses, le préfet informera le ministère de l'Intérieur qu'il n'y a aucune trace de ces objets dans les archives du camp ${ }^{11}$.

9. Arch. dép. de la Vienne, 104W167, fiches des internés.

10. Arch. dép. de la Vienne, 1W2761, rapport du commissaire de police judiciaire au commissaire principal, 3 septembre 1945 .

11. Arch. dép. de la Vienne, 1W2761, rapport du préfet au ministère de l'Intérieur, 14 janvier 1949. 
D'autres faits sont constatés après enquête, comme des fausses factures à une entreprise de transport, des travaux effectués chez le directeur aux frais du camp, etc. Le colonel Blanchard, directeur du camp, est suspendu (avant d'être mis à la retraite), ainsi que le gestionnaire et le chef du personnel (qui n'est autre que le fils du directeur). Nous n'avons pas étudié la suite de l'affaire en profondeur, mais nous savons qu'en 1951, des originaux de la comptabilité sont introuvables, alors que la Trésorerie générale adresse des " ordres de reversement " à l'ancien gestionnaire ${ }^{12}$.

\section{Les " Commandos "}

\section{Conditions d'emploi des prisonniers de guerre}

L'autorisation de l'utilisation des prisonniers de guerre, pour la reconstruction, mais également pour la production, est définie par la Convention de Genève. Dans le chapitre 2 , section 3 , il est précisé que "la puissance détentrice assumera l'entière responsabilité de l'entretien, des soins, du traitement et du paiement des salaires des prisonniers de guerre travaillant pour le compte de particuliers " (article 28). La durée journalière de travail, y compris les trajets aller et retour, " ne sera pas excessive et ne devra, en aucun cas, dépasser celle admise pour les ouvriers civils de la région employés au même travail. Il sera accordé à chaque prisonnier un repos de vingt-quatre heures consécutives chaque semaine, de préférence le dimanche " (article 30). Le " travail prohibé " (chapitre 3) n'est pas très clairement défini : " il est interdit d'employer des prisonniers de guerre à des travaux insalubres ou dangereux " (article 32).

Le ministère du Travail et de la Sécurité Sociale édite une brochure destinée aux mairies. Le but de l'utilisation des prisonniers est clairement exprimé : "Faites relever vos ruines par ceux qui en sont responsables. Faites embellir vos cités par ceux qui voulaient les détruire. Faites travailler les prisonniers ennemis ${ }^{13}$. "S'adressant directement aux municipalités, le ministère souligne les avantages :

"Songez à tous les travaux d'entretien et d'aménagement différés faute de main-d'œuvre et de crédits. Vous avez l'occasion, grâce aux prisonniers allemands, de les faire exécuter à des conditions exceptionnellement avantageuses pour votre commune et pour le pays. Ne la négligez pas. "

Pourquoi ne pas les faire travailler uniquement à la reconstruction? La raison est évidente :

"Ces prisonniers ne peuvent tous être immédiatement utilisés par le ministère de la Reconstruction faute de matériaux en quantités suffisantes. "

12. Arch. dép. de la Vienne, 104W145, rapport du préfet au ministère de l'Intérieur, 3 février 1951.

13. Arch. dép. de la Vienne, 1W1427, brochure éditée par le ministère du Travail et de la Sécurité Sociale. 
Et comme "ils ne doivent, à aucun prix, rester oisifs dans les dépôts militaires ", la seule solution est de les mettre au travail par petits commandos organisés dans toutes les communes de France où ils seront employés aux innombrables travaux de voirie ou de fossés et aux projets d'aménagement laissés en suspens depuis trop longtemps faute de maind'œuvre. La demande de prisonniers, par les maires, doit se faire auprès du préfet, qui transmet à l'inspecteur divisionnaire du Travail. Le commando doit comprendre un minimum pour cuisiner. Les prisonniers y sont enfermés la nuit, et doivent être gardés le jour par des ouvriers ou des chefs d'équipe. Restent les coûts à assumer pour les mairies. Ils se répartissent entre les frais d'alimentation, de logement et de garde, et le salaire. Ce dernier est de 10 francs par jour, versés au dépôt militaire (au compte du prisonnier), mais "l'État remboursera la moitié de ces frais à la commune ". Pour l'emploi de prisonniers chez des particuliers, le maire doit avant tout veiller à ce que sa commune ne comporte pas de chômeurs français. Le coût est plus important, car aux frais estimés pour les communes, s'ajoute "l'indemnité compensatrice ", versée au ministère du Travail.

Le directeur de l'Office régional du Travail établit, pour le préfet, un exemple mensuel financier pour l'emploi d'un prisonnier ${ }^{14}$. Un ouvrier agricole touche 1898 francs. Pour un prisonnier, l'employeur doit régler les frais d'alimentation (690 francs), de garde et d'entretien (300 francs) et le salaire du prisonnier (260 francs). La différence entre l'emploi d'un ouvrier agricole et d'un prisonnier est donc de 648 francs : c'est ce qu'on appelle "l'indemnité compensatrice". Elle permet d'éviter l'abus de l'emploi des prisonniers, qui pourrait pénaliser la main-d'œuvre française. Si les prisonniers logent ou mangent dans les commandos municipaux, l'employeur verse une somme équivalente aux frais au receveur municipal. Parodi, ministre du Travail, signale toutefois que les employeurs profitent d'un avantage conséquent :

" Je vous rappelle que l'emploi des prisonniers de guerre ne donne pas ouverture au versement des différentes charges sociales incombant normalement à tout employeur de main-d'œuvre française et qu'il y a là pour l'employeur de P.G. un premier avantage qui compense déjà l'infériorité de rendement ${ }^{15}$."

\section{Une main-d'œuvre surtout employée dans l'agriculture}

Localement, il reste à utiliser efficacement, avec des règles précises, cette main-d'œuvre nouvelle. Le préfet donne certaines indications au Comité Départemental de Libération :

14. Arch. dép. de la Vienne, 1W1427, lettre du directeur de l'Office régional du Travail au préfet, 5 juillet 1945 .

15. Arch. dép. de la Vienne, 1W1427, lettre du ministère du Travail et de la Sécurité Sociale aux directeurs départementaux du Travail et de la Main-d'œuvre, 24 mars 1945. 


\begin{abstract}
" D'après les services de la Main-d'œuvre, le placement des prisonniers de guerre chez les cultivateurs ne fait aucune difficulté; jusqu'ici 299 prisonniers ont été placés dans la culture, et le service a encore 788 demandes qu'il n'a pu satisfaire [...]. En fait, les cultivateurs se groupent eux-mêmes dans une même commune ou dans des communes voisines, se procurent un local (locaux ou dépendances inoccupés) et indiquent au Service de la Main-d'œuvre le nombre d'hommes dont ils ont besoin. Les commandos ainsi constitués doivent comprendre au moins six hommes. Après signatures des contrats, les prisonniers sont envoyés au lieu d'emploi où ils sont gardés par un ou deux militaires qui assument la responsabilité de la garde pendant la nuit et sont nourris, à tour de rôle, par les employeurs. Le service estime que les risques d'évasion de jour, lorsque les travailleurs sont dispersés dans les fermes qui les occupent, sont insignifiants, et les employeurs ne protestent pas contre cette responsabilité ${ }^{16}$."
\end{abstract}

Au 12 juin 1946, 2821 prisonniers de guerre sont employés dans la Vienne $^{17}$. La répartition est la suivante : 2096 dans l'agriculture, 261 dans les travaux publics, 134 dans la reconstruction, 101 dans les mines ou carrières, 97 dans les forêts et bois, 41 dans les industries mécaniques, 9 dans la sidérurgie, et 82 dans des activités diverses.

Au 19 septembre 1946, le nombre de prisonniers au travail est de 3990, dont 2460 pour l'agriculture et 721 pour la Reconstruction. Les communes utilisant des prisonniers les emploient souvent à la réfection des chemins, mais également aux travaux d'aménagement des lieux publics, comme les rénovations de mairies, écoles, ou champs de foire.

\title{
Les relations entre les autorités, la population locale et les prisonniers
}

Quels sont les problèmes rencontrés vis-à-vis de cette main-d'œuvre? Il faut tout d'abord souligner que les évasions sont peu nombreuses : entre janvier et juin 1946, on comptabilise 54 évadés, dont 36 ont été repris. Le commandant régional des prisonniers de guerre rappelle d'ailleurs que l'employeur doit payer 1500 francs de prime pour chaque prisonnier repris.

L'éloignement entre le local qui abrite les P.G. et leur lieu de travail pose parfois problème. Certains cultivateurs, comme à Tercé, demandent au préfet "l'autorisation de loger, chacun chez soi et sous sa responsabilité, les prisonniers allemands mis à leur disposition ${ }^{18}$ ". Le motif est la distance " entre la plupart des exploitations et le bourg de Tercé où est installé le Commando " et qui " varie entre deux et quatre kilomètres, et le temps passé en chemin par le prisonnier et l'homme qui l'accompagne représente de trois à quatre heures de travail ». Cette confiance vis-à-vis des Allemands est due au fait que " jusqu'ici les prisonniers employés à Tercé ont donné

16. Arch. dép. de la Vienne, 1W1426, lettre du préfet au C.D.L., 24 avril 1945.

17. Arch. dép. de la Vienne, 1W1427, rapport de De Franclieu au préfet, 12 juillet 1946.

18. Arch. dép. de la Vienne, 1W1426, lettre de cultivateurs de Tercé au préfet, 10 juillet 1945. 
satisfaction ". Le maire soutient les onze cultivateurs signataires. Mais les consignes sont strictes, et le commandant régional des P.G. ne transige pas :

"Ceux-ci doivent obligatoirement coucher la nuit groupés dans un local strictement verrouillé avec une fenêtre grillée, interdisant toute sortie et sous surveillance immédiate d'un gardien responsable du commando ${ }^{19}$. "

Pourtant, l'année suivante, ces consignes ne sont pas toujours suivies. D'après la gendarmerie de l'Isle-Jourdain,

" les communes de Adriers, Millac, Mouterre et Queaux ne détiennent aucun siège de commando de prisonniers de l'Axe. Dans chaque commune ces derniers travaillent dans les fermes et couchent chez leurs employeurs. Aucune mesure de sécurité n'est prise. Il n'est pas rare de les trouver seuls sur les routes se rendant à leur travail ou à bicyclette dans les communes voisines où ils se rendent pour faire des commissions même à des heures avancées de la nuit ${ }^{20}$."

Un débat au Conseil général porte sur la concurrence de la main-d'œuvre allemande, dans des communes où des Français sont au chômage ${ }^{21}$. Il est décidé d'un accord avec le préfet, pour que la Direction des prisonniers de guerre, avant de donner satisfaction aux demandes des cultivateurs, recueille l'avis du maire.

Il est à noter que la présence des Allemands déclenche par deux fois une violence meurtrière. En juin 1945, à trois semaines d'intervalle, et à quelques kilomètres de distance, ce sont douze puis six prisonniers allemands qui sont assassinés. Les douze premiers avaient disparu, en pleine nuit. Ils sont retrouvés dans une carrière abandonnée. D'après le sous-préfet de Châtellerault,

" les prisonniers ont été conduits sur place où ils ont été abattus, les uns par arme à feu, les autres par coups violents ayant amené l'enfoncement de la boîte crânienne. Deux d'entre eux avaient les mains liées derrière le dos par un fil de fer ${ }^{22}$."

Les six autres sont tués par une grenade lancée la nuit dans leur baraquement. Dans une commune voisine, cinq agriculteurs employant des Allemands reçoivent des menaces. Les renseignements généraux donnent quelques noms de suspects, mais le préfet juge qu'aucun fait n'est clairement établi et aucune arrestation n'a lieu. La population réprouve ces actes violents mais le commissaire de police judiciaire constate qu'il se " heurte souvent à des témoins qui déclarent qu'ils savent quelque chose mais qu'ils ne veulent rien dire ${ }^{23}$ ".

19. Arch. dép. de la Vienne, 1W1426, lettre du commandant régional de P.G. au préfet, 18 juillet 1945 .

20. Arch. dép. de la Vienne, 1W1427, rapport du commandant de la brigade de l'IsleJourdain, 16 mars 1946.

21. Arch. dép. de la Vienne, 1W1427, extraits du P.V. des délibérations du Conseil général, 14 et 17 juin 1946.

22. Arch. dép. de la Vienne, 1W3212, rapport du sous-préfet de Châtellerault au préfet, 27 juillet 1945.

23. Arch. dép. de la Vienne, 1W3212, rapport du commissaire de police judiciaire au commissaire principal, 15 novembre 1945. 
Comme nous l'avons souligné, l'histoire du camp de la Chauvinerie est particulière, par le type et le nombre d'internés, sa mortalité, et les affaires de détournement et d'abus. L'affaire dure plusieurs années et les condamnations sont faibles : le directeur du camp est condamné à reverser seulement le lait qu'il a détourné, à raisons de trois litres par jour. Son fils est amnistié après avoir été condamné à deux mois de prison pour vol d'habits de détenu. Le tribunal inflige 8 mois de prison au gestionnaire et un an de sursis et 25000 francs d'amende à deux marchands de légumes. Ces trois derniers doivent également verser 500000 francs au Trésor public ${ }^{24}$. L'histoire de ce "scandale " mériterait davantage de recherches, tout comme la provenance des internés, pour lesquels il faudrait sans doute consulter les archives des camps de provenance pour en savoir davantage sur leur motif d'internement.

Par une circulaire du ministère du Travail du 15 avril 1947, les Allemands des commandos ont la possibilité de devenir " travailleurs libres". Une " commission de criblage " accepte ou non les demandes. Les rapatriements se font selon des critères politiques, d'âge ou de situation familiale. Ainsi sont favorisés les " opposants au nazisme " et les plus de $50 \mathrm{ans}^{25}$. En mai 1948, 541 Allemands restés dans la Vienne, sont devenus " travailleurs libres ${ }^{26}$ ", dont 389 dans l'agriculture et 62 dans le bâtiment et les travaux publics. Ils se disent " anti-nazis et républicains ", mais la police se méfie :

" Il semble cependant difficile de déterminer si leurs allégations sont exactes étant donné qu'ils font surtout figure d'opportunistes et que la plupart sont des jeunes âgés de 20 à 30 ans, qui ont certainement subi une profonde influence de la propagande hitlérienne ${ }^{27}$."

Ils souhaitent rester en France, et même faire venir leur famille. La preuve en est qu'après un congé d'un mois accordé à chacun, "98 \% des permissionnaires sont revenus à leur travail ». De plus,

" les prisonniers de guerre allemands transformés sont en général bien considérés de leurs employeurs, notamment à la campagne, en raison de l'assiduité au travail, de la docilité et de la discipline dont ils font preuve."

Le seul bémol est le contact avec les autres Français :

« Ils vivent en dehors de la population française et ne se réunissent qu'entre eux les dimanches et jours de fête, soit chez les employeurs, soit dans les cafés, qu'ils ne fréquentent d'ailleurs pas de façon assidue."

La principale raison qui pousse les Allemands à vouloir travailler en France est la mauvaise situation en Allemagne :

24. La Nouvelle République, 27 janvier 1949.

25. Arch. dép. de la Vienne, 1W243, circulaire du ministère du Travail, 15 avril 1947.

26. Arch. dép. de la Vienne, 1W3212, rapport du chef des Renseignements généraux au directeur des Renseignements généraux à Paris, 22 mai 1948.

27. Idem. 
" Les divers prisonniers de guerre travailleurs qui ont été interrogés à leur retour sont unanimes à déclarer que l'existence en Allemagne est très difficile. D'après eux, le travail ne manquerait pas mais les salaires trop bas ne permettraient pas de vivre convenablement. De ce fait, ces étrangers ne désirent pas partir définitivement avant un an, délai qui leur paraît un minimum pour que la situation se soit améliorée sensiblement dans leur pays d'origine ${ }^{28}$."

$\mathrm{Si}$, à quelques exceptions près, les habitants de la Vienne ont " accepté " la présence allemande dans leur département, nous avons pu remarquer dans nos recherches qu'elle reste assez indifférente au sort funeste des internés du camp de la Chauvinerie. La population est marquée par les années d'occupation, et se préoccupe davantage des restrictions qui la touchent encore après la fin de la guerre. Mais en ce qui concerne le camp de la Chauvinerie, ce sont bien des membres de l'administration publique qui ont abusé des biens des prisonniers, et qui n'ont pas réagi à la mortalité élevée des internés.

28. Arch. dép. de la Vienne, 1W243, rapport mensuel du préfet, février 1948. 


\section{RESUME}

De février à novembre 1945, le camp de la Chauvinerie à Poitiers accueille plusieurs milliers d'internés allemands. Pour une grande majorité, ce sont des civils, évacués des terrains de bataille au fur et à mesure de la progression des Alliés dans l'Est de la France et en Allemagne. Enfants, vieillards, hommes et femmes y sont retenus. L'histoire de ce camp est marquée par ce qu'on a appelé le " scandale du camp de la Chauvinerie ". En effet, le taux de mortalité y est très fort, et touche notamment les enfants en bas âge. Le Directeur et le gestionnaire doivent répondre d'accusations de détournements de biens et d'aliments. Il leur est reproché notamment des échanges entre gardiens et internés, un détournement d'aliments, et la saisie et la distribution des biens des internés. L'histoire remonte jusqu'au gouvernement qui veut déterminer les responsabilités.

Une autre présence allemande marque également l'histoire du département après la guerre. La France utilise les prisonniers de guerre allemands pour la reconstruction. Près de 4000 prisonniers, présents dans le département de la Vienne, sont répartis dans nombre de communes, pour certes reconstruire, mais surtout dans l'agriculture où l'on essaie de pallier au manque de main-d'œuvre. La Convention de Genève définit les droits des prisonniers et des " puissances détentrices " de ces travailleurs. En 1948, lorsque les prisonniers ont la possibilité de rentrer en Allemagne, plus de 500 décident de rester dans la Vienne avec le statut de " travailleurs libres ". Comment ont-ils été utilisés? Quels rapports ont-ils eu avec les Français? Quel traitement leur a t-on réservé?

\section{ABSTRACT}

From February to November 1945, the camp of the Chauvinerie in Poitiers welcomes several thousands interned German. Most of them, are civilians evacuated from battlefields according to the progression of the Allies in the East of France and in Germany. Children, old persons, men and women are kept there. The story of this camp is marked by the "scandal of the camp of the Chauvinerie". In fact, the mortality is very strong, and strikes notably the very young children. The Director and the administrator were accused for having misappropriated personnal estate and food. They are accused of having done exchanges between watchmen and prisoners, misappropriation of food, and the seizure and the distribution of the internees' goods and chattels. The history dates back to the government willing to find out the responsibilities.

Another German presence also marks the department's story after the war. France uses the German prisoners of war for the reconstruction. Close to 4000 prisoners, being in Vienne's department, are divided up in several villages, indeed to rebuild, but above all in agriculture to make up for the lack of workers. The Geneva Convention defines the rights of the prisonners and the countries welcoming these workers. In 1948, when the prisonners have the opportunity to go back to Germany, more than 500 decide to stay in Vienne's department with the status of "free workers". How were they used? Which relations did they have with the French? Which treatment do they suffer from? 



\section{Approches régionales contemporaines}


